\title{
Clinicopathological Features of Ophthalmic Neoplasms Arising in the Setting of Xeroderma Pigmentosum
}

\author{
Maria J. Suarez ${ }^{\mathrm{a}}$ Roxana Rivera-Michlig ${ }^{c}$ Sander Dubovy ${ }^{\mathrm{d}}$ \\ Fausto J. Rodriguez ${ }^{a, b}$ \\ a Department of Pathology, bSidney Kimmel Comprehensive Cancer Center, and 'Wilmer Eye Institute, Johns \\ Hopkins University School of Medicine, Baltimore, Md., and d Bascom Palmer Eye Institute, University of Miami, \\ Miami, Fla., USA
}

\section{Key Words}

Xeroderma pigmentosum · Melanoma Squamous cell carcinoma - Basal cell carcinoma - Eyelid - Ocular surface . Immunohistochemistry

\section{Abstract}

Background: Patients with xeroderma pigmentosum (XP) are strongly predisposed to the development of numerous cutaneous cancers. However, the extent of ocular pathology in these patients has not been adequately studied. Methods: We conducted a retrospective study of tumors involving the ocular surface and ocular adnexa from 6 XP patients. Histopathological evaluation and immunohistochemistry was performed using antibodies directed against the most common mutated proteins in XP (XPA, XPC, and XPD). Results: Patients included 4 males and 2 females with a mean age of 20.8 years (range 10-31) who met the clinical criteria for XP and were found to have a total of 13 neoplasms involving the ocular surface and adnexal skin; 6 squamous cell carcinomas (SCC), 3 cases of conjunctival intraepithelial neoplasia, 2 malignant melanomas, 1 basal cell carcinoma, and 1 atypical fibroxanthoma. Complete XPD loss was present in two tumors from 1 patient, suggesting a germline defect, and in the invasive component of an SCC from a second patient, suggesting a somatic alteration. No clear pattern of loss for XPA or XPC was evident. Conclusions: Our study outlines our early experience with the pathology of ocular neoplasms in XP patients. These findings deserve further exploration with genetic studies and additional patients.

(c) 2015 S. Karger AG, Basel

\section{Introduction}

Xeroderma pigmentosum (XP) is a rare genetic disorder characterized by severe hypersensitivity to ultraviolet (UV) light due to mutations in genes responsible for DNA repair, specifically nucleotide excision repair (NER) [1]. $\mathrm{XP}$ and other related disorders are caused by autosomal recessive mutations, resulting in a diverse and wide variety of pathologies affecting most systemic and neural tissue organs, depending on the specific alteration $[2,3]$. Other disorders from the NER-related family include De Sanctis-Cacchione syndrome ('XP with neurologic complications'), XP variant (XP-V), Cockayne syndrome (CS), cerebro-oculo-facio-skeletal syndrome, mild UV-sensitive syndrome, trichothiodystrophy, and XP/CS complex [2]. In XP in particular, repair of UV-associated damage is impaired. UV radiation leads to dipyrimidine nucleo-

\section{KARGER}

E-Mail karger@karger.com

www.karger.com/oop
(C) 2015 S. Karger AG, Base

2296-4681/15/0022-0112\$39.50/0
Fausto J. Rodriguez, MD

Department of Pathology, Johns Hopkins Hospital

Sheikh Zayed Tower, Room M2101

1800 Orleans Street, Baltimore, MD 21231 (USA)

E-Mail frodrig4@jhmi.edu 
Fig. 1. Overview of the NER system as the genetic basis for XP and related disorders. TCR removes damaged DNA from genes that are actively transcribed, while GGR works in the remaining genome. In GGR, the DNA damage by UV radiation leads to the formation of cyclobutane pyrimidine dimers (CPDs) or 6-4 photoproducts (PPs), which are recognized by XPC proteins. Meanwhile in TCR, DNA damage blocks RNA polymerase II function. Pathways overlap after initial damage recognition, in a process involving XPB, XPG, $\mathrm{XPA}$, and replication protein A (RPA). Finally, XPF and XPG remove the damaged regions by making incisions of $\sim 30$ nucleotides, followed by DNA synthesis to fill the gaps.

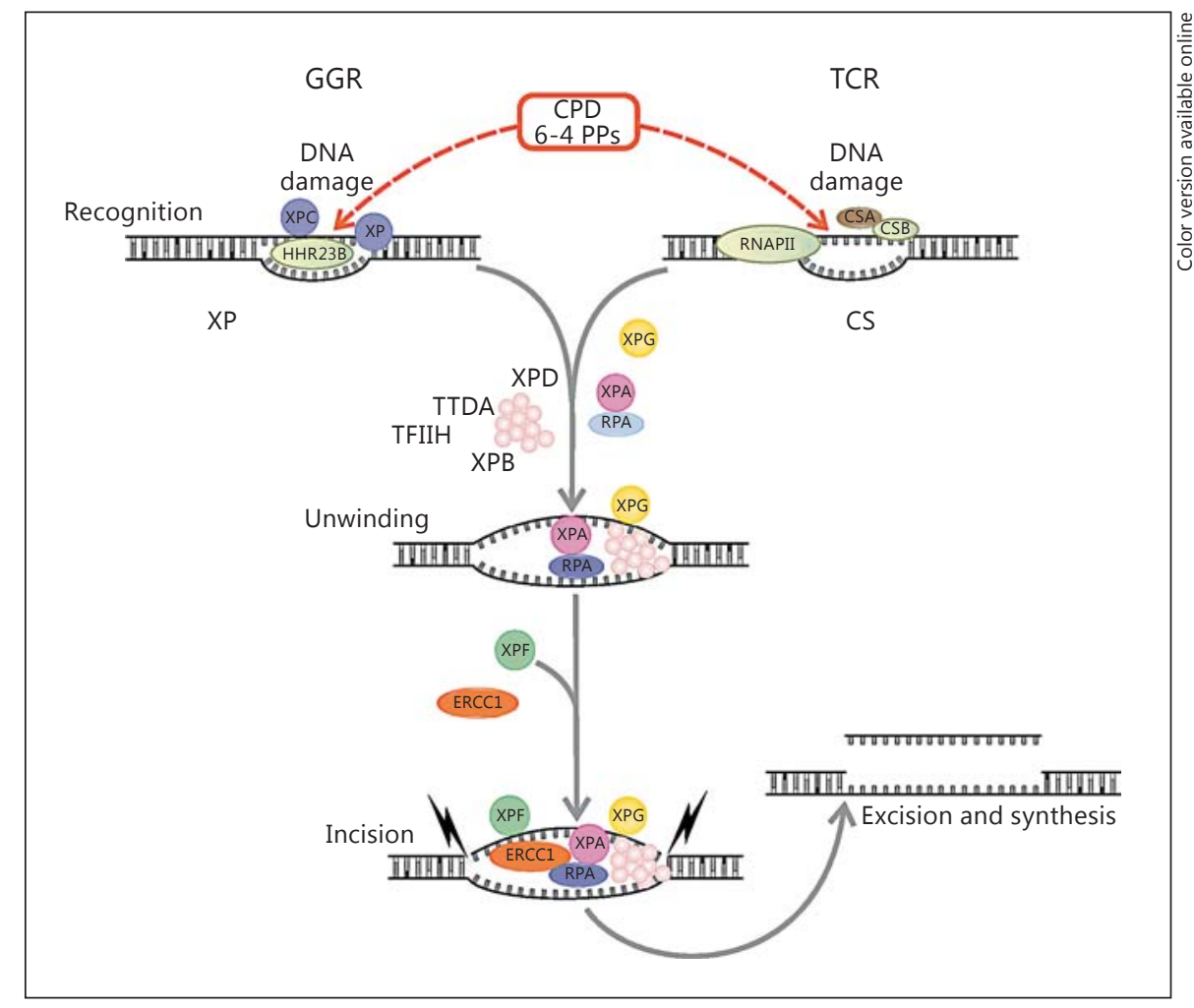

tide products, which are recognized by the NER pathways (fig. 1). Unrepaired products in XP during replication lead to errors resulting in the incorporation of a wrong nucleotide, resulting in characteristic C-to-T or CC-to-TT mutations of UV damage. NER involves two separate but converging pathways: global genome repair (GGR), recognizing damage in DNA that is not being transcribed (99\%), and transcription-coupled repair (TCR), which is recognized when RNA polymerase stops in transcriptionally active genes (1\%). There is little redundancy in these pathways, and therefore, alterations in individual components result in clinical syndromes. In general, defects in the GGR components (XPC and XPE) lead to pure XP with no neurologic abnormalities, while defects in TCR lead to CS and severe neurologic abnormalities. Complex and variable phenotypes result from abnormalities in the common repair pathway. Alterations in eight different genes have been associated with XP; XPA, XPC, and XPD are the most common and prevalent pathogenic variants in the United States and Europe [3-5]. Genes known to be associated with XP are summarized in table 1 . Given the important role of the proteins encoded by these genes in DNA repair, that most of these disorders are autosomal recessive and lead to complete protein loss, and that the mutations may occur in many sites of different genes, immunohistochemistry represents an attractive technique with the potential to identify the possible aberrant gene in tissues from these patients.

Overall, clinical manifestations of XP are commonly seen in sun-exposed areas such as skin, mucous membranes, and eyes, including the ones seen after a few minutes of sun exposure (blisters), pigmented freckle-like lesions, ocular abnormalities, and a well-known significant increased risk of developing neoplasia in these areas [3]. Ocular manifestations in patients with XP include photophobia, conjunctivitis, ectropion, secondary exposure keratitis with subsequently corneal opacification and vascularization, pterygium, and neoplasia $[3,6,7]$. In a recent study of 87 XP patients, $91 \%$ had ocular manifestations, and a history or clinical evidence of ocular surface neoplasia was present in $10 \%$ [6]. The spectrum of neoplasia affecting the ocular surface and adnexa of patients with XP has not been as frequently studied as cutaneous manifestations, but includes squamous cell carcinoma (SCC), basal cell carcinoma (BCC), and malignant melanoma (MM). A preneoplastic spectrum known as conjunctival intraepithelial neoplasia (CIN), classified as mild, moderate, or severe depending on the depth of conjunctival epithelial 
Table 1. Genetic alterations associated with XP (modified from Kraemer et al. [3])

\begin{tabular}{|c|c|c|c|c|}
\hline Gene & Molecular pathway & Proportion & Phenotype & Geography \\
\hline$X P A$ & Common repair pathway & $25 \%$ & $\begin{array}{l}\text { XP with mild-to-severe neurologic } \\
\text { abnormalities }\end{array}$ & $\begin{array}{l}\text { Common in Japan, rare in the } \\
\text { US and Europe }\end{array}$ \\
\hline ERCC3 (XPB) & Common repair pathway & Rare & $\begin{array}{l}\text { XP/CS } \\
\text { TTD } \\
\text { XP with mild neurologic } \\
\text { abnormalities }\end{array}$ & \\
\hline$X P C$ & GGR & $25 \%$ & Nonneurologic & \\
\hline ERCC2 (XPD) & GGR/TCR (at the junction) & $15 \%$ & $\begin{array}{l}\text { Complex manifestations (XP, CS, } \\
\text { XP/CS, XP/TTD, COFS) }\end{array}$ & \\
\hline$D D B 2(X P E)$ & GGR & Rare & Nonneurologic & \\
\hline ERCC1 & Common repair pathway & Rare & COFS & $\begin{array}{l}\text { One patient reported (born to } \\
\text { nonconsanguineous white } \\
\text { Italian parents) [6] }\end{array}$ \\
\hline$P O L H(X P V)$ & $\begin{array}{l}\text { Technically, a polymerase } \\
\text { not part of NER }\end{array}$ & $21 \%$ & XP with no neurologic abnormalities & \\
\hline
\end{tabular}

dysplasia, can also present secondary to sun exposure with this underlying genetic disorder $[3,4]$. Other skin tumors including keratoacanthoma and fibrosarcoma [3] as well as angiosarcoma of the tongue have been additionally reported [8]. Atypical fibroxanthoma (AFX) is a benign tumor seen in previously sun-damaged skin of the head and neck in the elderly $[9,10]$. Shao et al. [10] have reported the first case in a 6-year-old African American boy with XP with simultaneous conjunctival AFX and SCC.

To clarify the spectrum of neoplasia affecting the ocular structures and adnexa in XP patients specifically, we present our experience with the clinical, pathologic, and immunohistochemical features of neoplasms involving the ocular surface and/or adnexa in these patients and perform a literature review.

\section{Materials and Methods}

Clinical records from 6 patients with XP and neoplasms involving the eye and/or ocular adnexa with pathologic material ( 3 from the Wilmer Eye Institute at Johns Hopkins University and 3 from the Bascom Palmer Eye Institute at the University of Miami) were studied. All histologic sections from tumors involving the ocular surface and ocular adnexa stained with H\&E were reviewed. Immunohistochemical studies were performed using antibodies against the most common proteins affected by XP mutations XPA (Mouse monoclonal, 1:50; GeneTex, Irvine, Calif.), XPC (Mouse monoclonal, 1:100; GeneTex), and XPD (Rabbit polyclonal, 1:500; GeneTex). In brief, slides were deparaffinized and antigen retrieval was performed with sodium citrate. Slides were incubated with the primary antibody overnight at room temperature, washed, and incubated for $1 \mathrm{~h}$ with a biotinylated secondary antibody. Scoring of nuclear immunoreactivity was performed using a 3-tiered scale: $2=$ moderate to strong staining, $1=$ weak/focal staining, and $0=$ negative staining. Complete protein loss in multiple samples and both neoplastic and nonneoplastic tissues was interpreted as suggestive of a germline mutation, while protein loss in neoplastic tissues would only be interpreted as suggestive of somatic protein dysfunction.

\section{Results}

\section{Case Histories}

Clinical and pathologic features of the tumors are summarized in table 2 and illustrated in figures 2-4. 
Table 2. Immunohistochemical studies for XP-associated proteins XPA, XPC, and XPD

\begin{tabular}{|c|c|c|c|c|}
\hline & Pathology & $\mathrm{XPA}$ & $\mathrm{XPC}$ & $\mathrm{XPD}$ \\
\hline \multirow[t]{2}{*}{ Patient 1} & In situ and superficially invasive conjunctival SCC & Positive & Positive & Positive \\
\hline & Well-differentiated SCC of the conjunctiva & Positive & Positive & Negative \\
\hline \multirow[t]{3}{*}{ Patient 2} & Malignant melanoma & Negative & Weak/focal & Weak/focal \\
\hline & Recurrent melanoma & Weak/focal & Weak/focal & Weak/focal \\
\hline & Nodular BCC on the lower eyelid & Negative & Positive & Positive \\
\hline \multirow[t]{2}{*}{ Patient 3} & CIN with moderate atypia & Negative & Weak/focal & Weak/focal \\
\hline & Well-differentiated SCC of the lower eyelid & Positive & Weak/focal & Positive \\
\hline Patient 4 & Moderately differentiated SCC of the lower eyelid & Positive & Positive & Positive \\
\hline Patient 5 & CIN with severe dysplasia & Negative & Positive & Negative \\
\hline
\end{tabular}

\section{Patient 1}

An 11-year-old African American male initially presented at age 3 with sun hypersensitivity associated with coarse skin, hypo- and hyperpigmented macules on his face, neck, upper chest, upper back, and bilateral upper arms. At age 7, he presented with severe photophobia, bilateral ocular injection, and bilateral nasal white conjunctival lesions initially diagnosed as pterygium. These lesions were excised and proved to be SCC. His visual acuity was 20/30 in the right eye and 20/50 in the left eye. At age 9 , the patient presented with a recurrence of SCC in the right eye that was treated surgically. At age 10, he developed SCC of the lower lip, which recurred. Recently, the patient also developed a SCC of the tongue.

\section{Patient 2}

A Sudanese male with a history of consanguinity of his parents and a sibling with XP developed eye pain, photophobia, irritation, and blurry vision at the age of 4 , with subsequent bilateral corneal opacities and a history of multiple skin cancers. He moved to the United States and, at age 20 , presented with a 1 -year history of discomfort in both eyes with associated photosensitivity for many years. His visual acuity in the right eye was 20/50, and count fingers was at 3 feet in the left eye. An external examination revealed multiple patchy, hyperpigmented areas of the skin. Slit-lamp examination was remarkable for scarred areas and hyperemic upper and lower lids as well as for bilateral lower-lid ectropion. Both eyes presented corneal opacities and neovascularization, more in the left than in the right. At age 25, both lower lids became inflamed, scarred, and thickened. One year later, a hemorrhagic exudative right anterior orbital mass was noted. This was excised and found to be MM with positive margins that recurred 1 year later and for which he underwent further palliative surgical excision. He later developed BCC of the lips and eyelids and SCC of the conjunctiva and cornea. Recently, the patient died secondary to a diffuse large B-cell lymphoma.

\section{Patient 3}

A 15-year-old female from the Central African Republic presented with a history of XP and multiple tumors on the face, including BCC and melanoma in addition to angiosarcoma of the tongue (the pathologic findings of the tongue neoplasm have previously been reported) [8]. The patient first presented at the age of 10 with no light perception vision in the right eye and 20/40 in her left eye. She has had no light perception in the right eye since age 4. She had complete corneal opacification in the right eye and partial corneal opacification and scarring in the left eye. She also presented lid margin keratinization and loss of lashes. At age 12, the patient presented CIN with severe atypia on her left eye. A couple of months later, the lesion recurred. Excision showed CIN with moderate atypia. One year later, she presented with an ulcerated right lower lid lesion that proved to be SCC and a new pigmented conjunctival lesion in her left eye. More recently, the patient developed melanoma in situ in the left shoulder and MM in the forehead. 

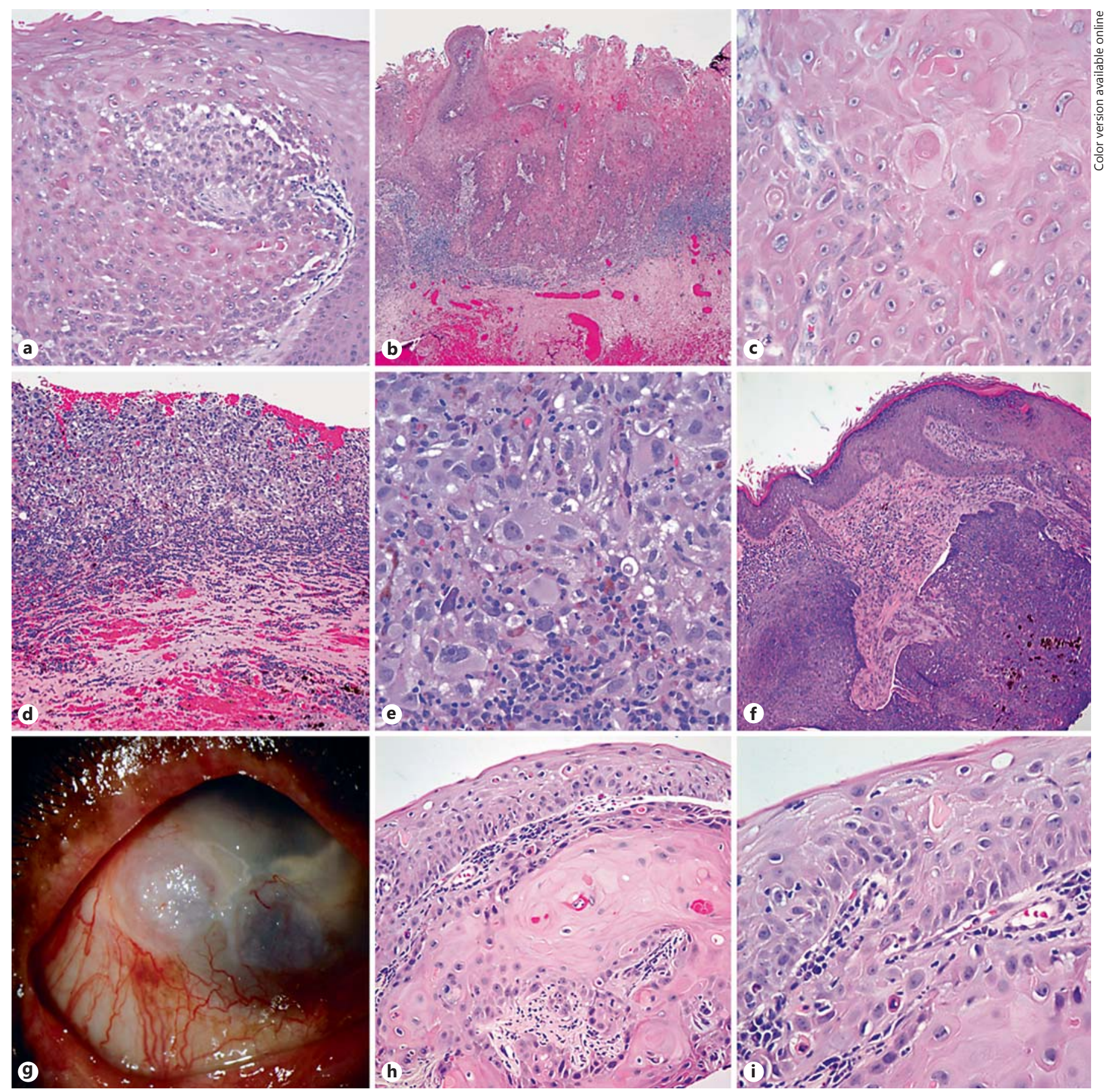

Fig. 2. Spectrum of neoplasms involving the eye and ocular adnexa in patients with XP. XP patients are predisposed to ocular surface squamous neoplasia including in situ (a) and frankly invasive SCCs of the conjunctiva (b, c). Patient 2 developed the whole spectrum of XP-associated neoplasia, including recurrent melanoma (d, e), nodular BCC of the eyelid (f), and striking conjunctival, keratinized limbal and conjunctival lesions (g), which histologically demonstrated in situ and superficially invasive SCC components (h, i). 

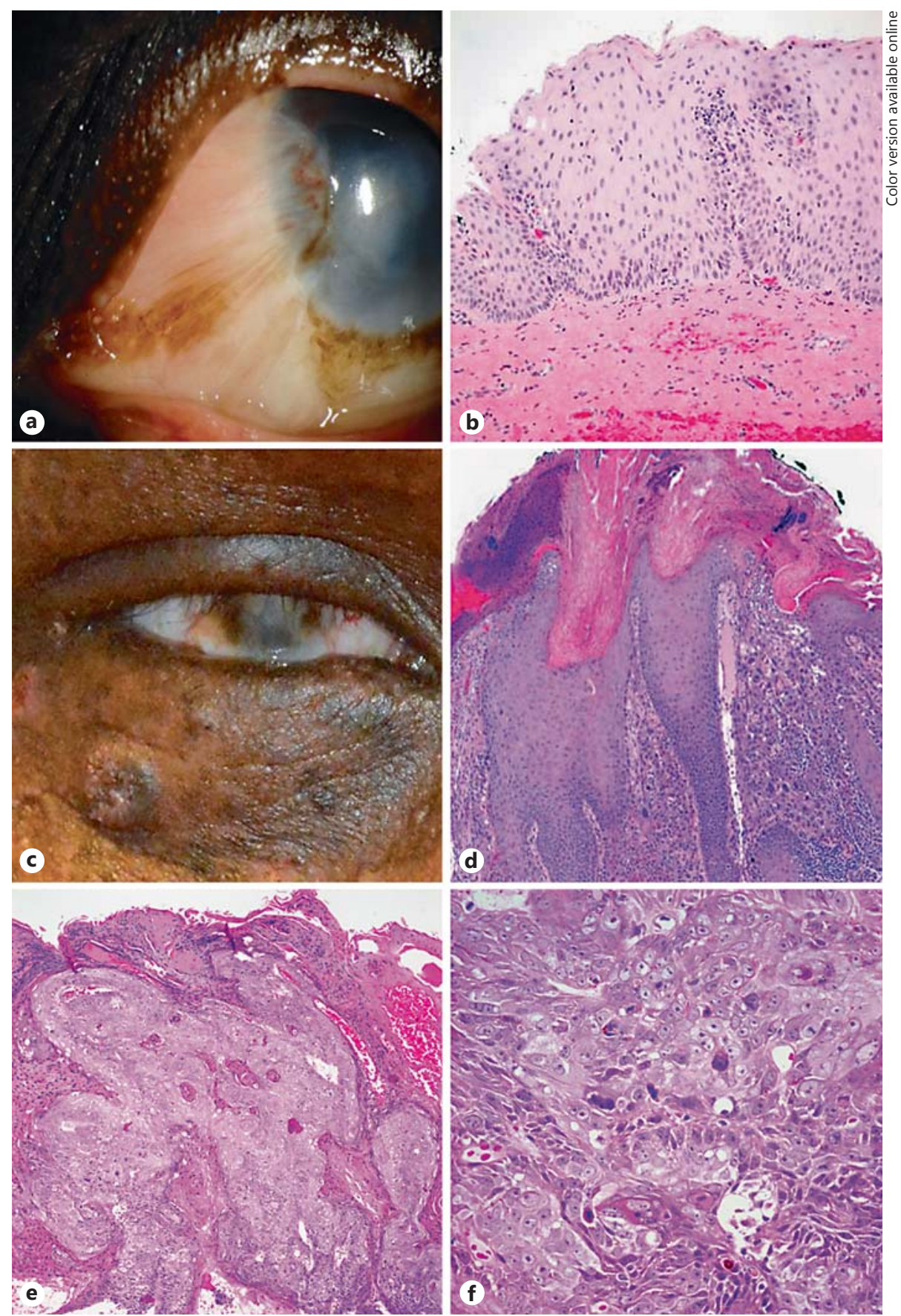

Fig. 3. Spectrum of differentiation of squamous neoplasia in XP. XP patients develop a variety of squamous lesions, including CIN with variable dysplasia $(\mathbf{a}, \mathbf{b})$ and welldifferentiated, highly keratinized eyelid SCCs (patient 3; c, d). A subset of tumors may be less differentiated and demonstrate increased pleomorphism $(\mathbf{e}, \mathbf{f})$.

\section{Patient 4}

A 31-year-old Hispanic male with XP presented at age 15 with a crater-like lesion at the right lower lid and was sent overseas to receive medical care. The lesion was excised and consistent with moderately differentiated SCC. No further clinical information could be retrieved due to limited data on his one-time visit to the US.

Ophthalmic Neoplasms in Xeroderma

Pigmentosum

\section{Patient 5}

A 26-year-old white Hispanic male with previous chronic sun exposure was diagnosed with XP at age 5 . The patient presented at age 12 with a large left lower-lid lesion concerning for malignancy that was excised with subsequent skin graft in his home country. The skin graft became infected, the lesion progressed leading to a blind 
Fig. 4. AFX in XP. This XP patient developed a fleshy mass in the conjunctiva (a). Histologic examination demonstrated significant pleomorphism, xanthic change, and an immunophenotype most supportive of $\operatorname{AFX}(\mathbf{b})$.
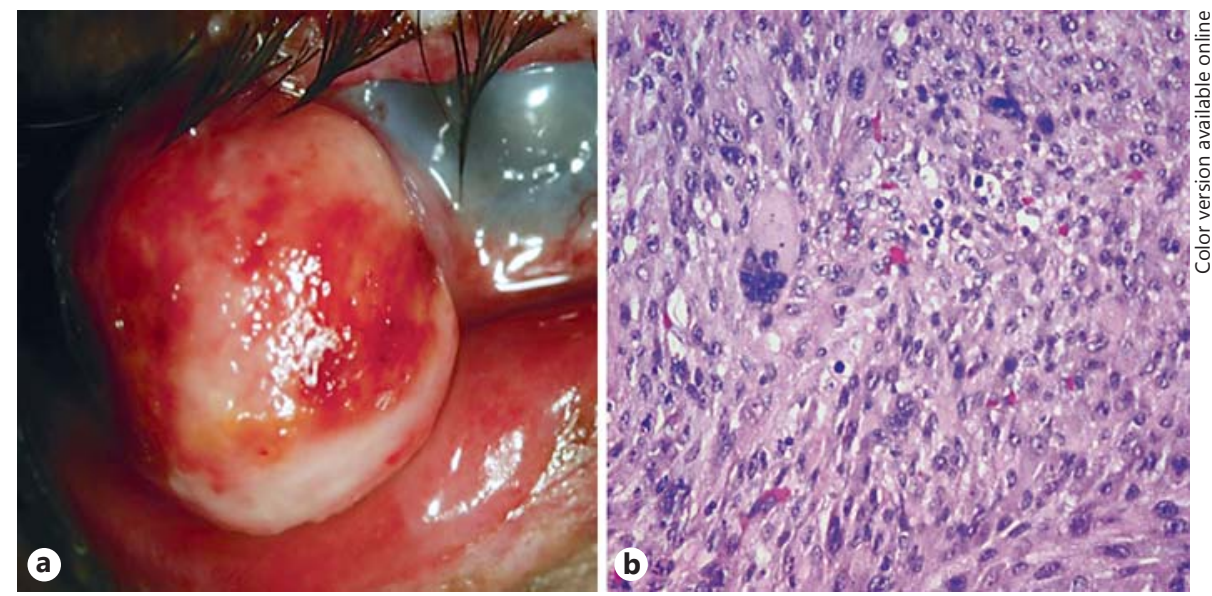

painful eye, and he was then transferred for eye enucleation and resection of part of his left alar wing of the nose. At age 13, the mother noticed enlarged lymph nodes in his right submental area and mandibular angle. Fine-needle aspiration of both areas were done and revealed level I lymph node with metastatic MM in one out of two lymph nodes $(3.7 \mathrm{~cm})$ and right superficial parotid plus infraparotid lymph nodes with metastatic MM in one out of two lymph nodes $(3.5 \mathrm{~cm})$. No primary melanoma was found, and the parents denied previous diagnostic biopsies. The patient received interferon and radiotherapy. Few years later, the patient presented with a pearly and ulcerated lesion in his right brow. At age 21, he presented with a white, friable right conjunctival lesion that was excised and proved to be CIN with severe dysplasia. One year later, the patient presented with a rapidly progressive and enlarging lesion in his right lower palpebral conjunctiva. This was excised and showed to be AFX. In the meantime, the patient has had multiple extraocular manifestations, including SCC, focal atypical melanocytic hyperplasia, hemangiomas, and numerous skin lentiginous lesions.

\section{Patient 6}

A 16-year-old white female first presented with severe photophobia and bilateral keratopathy secondary to sun exposure and diffusely dust, hyperpigmented, tan macules all over her skin as well as with numerous well-healed scars on the chest and back, including one hypertrophic scar on the left upper back. The patient developed a white, elevated and vascularized conjunctival lesion in her right eye that proved to be CIN with severe dysplasia. Recently, the patient presented with a $2-\mathrm{mm}$ pearly papule in her right lower eyelid that has not been biopsied.

\section{Pathology Summary}

We studied thirteen specimens from the 6 patients described above. Histologic examination revealed 3 patients with CIN: 1 with moderate and 2 with severe dysplasia. Four patients developed 6SCC: 3 arising from the conjunctiva ( 2 well differentiated and 1 in situ superficially invasive carcinoma) as well as 3 SCC of the lower eyelid (1 moderately, 1 well-differentiated, and 1 in situ carcinoma). One of the patients presented with an orbital melanoma that recurred, in addition to a nodular BCC in the lower eyelid. A single patient had an AFX arising from the conjunctiva.

\section{Immunohistochemical Findings}

Immunohistochemical stains per case are outlined in table 2. XPC immunoreactivity was preserved in all tumors from every patient, at least focally. XPD was negative in neoplastic and nonneoplastic cells in both tumors from patient 5 , in contrast to XPA and XPC, suggesting that the corresponding gene could be altered in this patient at the germline. XPD was also negative in an invasive SCC from patient 1 but not in an in situ SCC or conjunctival epithelium from the same patient, while both XPA and XPC were preserved, suggesting that it could represent a somatic alteration in XPD. XPA was negative in at least one tumor in 3 patients, but positive in other tumors from the same patient, suggesting it was not a germline defect. The nuclear scoring of the different tumors is illustrated in figure 5 .

\section{Discussion}

$\mathrm{XP}$ is an inherited rare autosomal recessive disease with unaffected heterozygotic carriers [6], which is a member of a spectrum of diseases with underlying associated NER mu- 


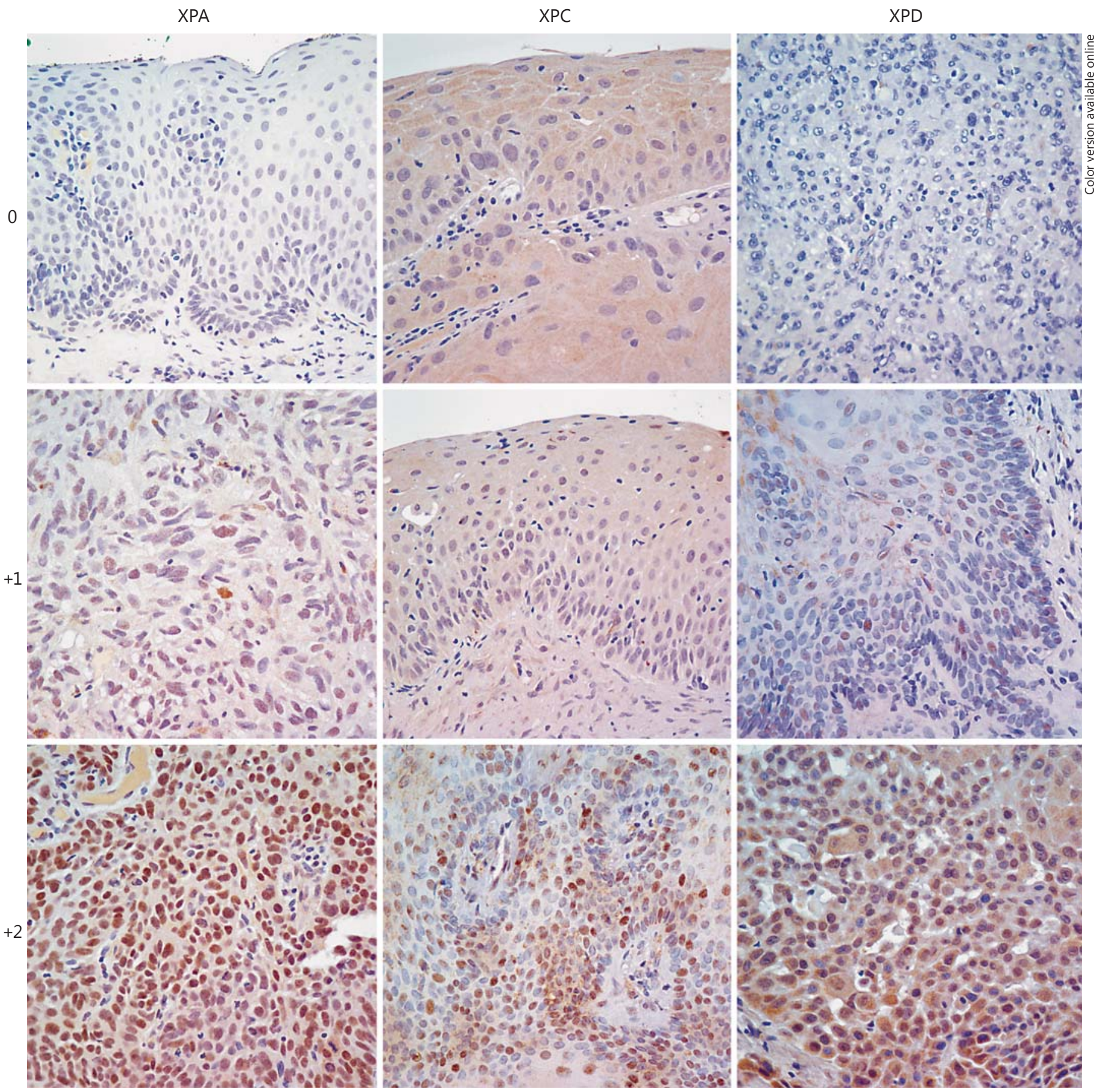

Fig. 5. Immunohistochemical scoring for XPA, XPC, and XPD in XP-associated ocular neoplasms. Nuclear immunohistochemical staining for XP-associated proteins was scored in a three-tiered scale: negative (0), weakly/ focal positive $(1+)$, or moderate/strongly positive $(2+)$.

tations first described by Moriz Kaposi in 1874 and interpreted by Cleaver as a deficient DNA excision repair process in XP cells $[1,2]$. The first case report of XP in the US was in 1878 by Taylor [11], followed by the first case re- ported in an African American in 1940 by King and Hamilton [12]. The ocular manifestations of XP patients was nicely reviewed by Reese and Wilber [13] in 1943, prior to our current understanding of the molecular pathogenesis. 
In the US and Europe, the prevalence of XP is approximately $1: 1,000,000$, and it is present in all races [14]; a higher prevalence is seen in Japan (1:22,000), North Africa, and the Middle East where associated parent consanguinity is relatively common. In Saudi Arabia, for example, a previous report found that consanguinity occurred in a third of the cases studied [15]. The exposure to sunlight in patients with XP leads to an early development of skin and mucous membrane neoplasia, including SCC, BCC, and melanoma. De Feraudy et al. [16] reported that UV-induced cases of neoplasia arise in the setting of specific XPC and XPE mutations and that XPB, XPD, XPF, and XPG defects are associated with progressive neurodegenerative and developmental disorders. Furthermore, XPA mutations may result in an overlap of these two scenarios.

In general, XP diagnosis is based on the patient history and clinical findings. Sunlight exposure in the context of these genetic DNA repair mutations manifest as cellular hypersensitivity to UV light, leading to an increased susceptibility and incidence of cutaneous basal and squamous cell malignancies and melanoma at an early age [3]. Symptoms may develop in the first couple of years of sun exposure, including the presence of hypo- and hyperpigmented macules seen in exposed areas as well as severe sunburns.

This study outlines our early experience with the pathology of ocular neoplasms in the context of XP. We studied 6 patients, 4 males and 2 females, with a mean age of 20.8 years who met the clinical criteria for XP and were found to have a total of 13 neoplasms involving the ocular surface and adnexal skin. Pathologic examination revealed 6 SCC, 3 CIN, 2 MM, 1 BCC, and 1 AFX (table 2). Our findings are similar to those reported in the literature: there is an increased risk present in this population for the early development of cutaneous tumors before the age of 20, and the mean age seen in our patients was 20.8 years $[3,10,16,17]$. The clinical presentation varies according to the extent of tissue damage, including impaired visual acuity, photophobia, and subsequent corneal opacity $[3,15,17]$. In our population, severe photophobia was present in 4 out of 6 patients studied. Furthermore, the presence of congested conjunctiva with associated pterygium-like growth lesions, conjunctival melanosis, lid freckles, and atrophic scarred skin lead to ectropion and symblepharon of the lower lid that can progress to neoplasia. In the interpalpebral zone of the limbus, there is approximately a 20 times higher UV light intensity, affecting the limbal stem cells and likely contributing to the development of tumors $[3,10,18,19]$.
The incidence of these conjunctival preneoplastic lesions was seen in a third of the cases (33\%), and one of them recurred after excision.

Additionally, we also described an increased prevalence of SCC that clinically presented as a white, flat lesion with irregular borders in the conjunctiva and as crater-like, ulcerated, and elevated mass in the eyelid with superficial invasion. Previous reports described SCC of the eye/orbit as an invasive tumor that may spread into the brain $[6,17]$. Periocular tumors can also arise in the setting of XP, and 1 patient in our study presented with recurrent orbital melanoma and $\mathrm{BCC}$ of the eyelid [3, 10, 17-19]. Interestingly, less common cutaneous neoplasia such as AFX has also been reported in the facial skin and lower lip in patients with XP, as well as 1 case affecting the conjunctiva in an African American 5 -year-old boy [10]. From our findings, we can also conclude that this neoplasia arises in sun-exposed areas, particularly in the lower palpebral conjunctiva, and presents as a solitary, well-circumscribed, and rapidly growing mass $[3,10]$.

We also complemented our clinical observations with immunohistochemical studies, taking into account our contemporary knowledge of the individual components mutated in XP patients. This approach using archival tissue from XP patients has been found valuable by others [16]. In our study, there was no indication that XPC genetic alterations were a culprit for the tumors examined. There was a suggestion that immunohistochemistry for XPA and XPD could be more useful in the study of ocular tumors affecting XP patients, particularly those with an invasive component [16]. However, given the limited number of patients studied, these findings deserve further exploration with formal genetic analysis and testing of additional patients. Refinement of immunohistochemical testing and scoring for XP pathway protein components has the potential to help in initial pathologic screens for patients with clinical suspicion and may become part of routine workup, particularly in centers that experience an increased number of encounters with such patients.

In summary, the spectrum of neoplasms involving the ocular surface and adnexa in XP patients is similar to that more commonly recognized to involve the skin in the setting of sun exposure. However, the increased frequency of ocular surface squamous neoplasia is an important ocular-specific complication occurring at an early age in these patients, which requires specialized and often lifelong care. The potential utility of immunohistochemical analysis for proteins encoded by XP genes may be helpful 
in a subset of cases, including the evaluation of possible germline and/or somatic alterations in XP-associated tumors. Our findings remain to be validated in the future in larger cohorts, but this case series approach could provide a basis for future investigation.

\section{Acknowledgments}

We have conducted this study with the support of Research to Prevent Blindness.

\section{Statement of Ethics}

The study was carried out in compliance with the guidelines for human studies and under Institutional Review Board approval allowing retrospective patient review, including informed consent when appropriate. No animal studies were performed.

\section{Disclosure Statement}

The authors have no conflicts of interest to disclose.

\section{References}

1 Cleaver JE: Cancer in xeroderma pigmentosum and related disorders of DNA repair. Nat Rev Cancer 2005;5:564-573.

2 Cleaver JE, Lam ET, Revet I: Disorders of nucleotide excision repair: the genetic and molecular basis of heterogeneity. Nat Rev Genet 2009; 10:756-768.

3 Kraemer KH, Lee MM, Scotto J: Xeroderma pigmentosum. Cutaneous, ocular, and neurologic abnormalities in 830 published cases. Arch Dermatol 1987;123:241-250.

4 Gupta N, Sachdev R, Tandon R: Ocular surface squamous neoplasia in xeroderma pigmentosum: clinical spectrum and outcome. Graefes Arch Clin Exp Ophthalmol 2011;249: 1217-1221.

5 Rivera-Begeman A, McDaniel LD, Schultz RA, Friedberg EC: A novel XPC pathogenic variant detected in archival material from a patient diagnosed with xeroderma pigmentosum: a case report and review of the genetic variants reported in XPC. DNA Repair (Amst) 2007;6:100-114.
6 Brooks BP, Thompson AH, Bishop RJ, et al: Ocular manifestations of xeroderma pigmentosum: long-term follow-up highlights the role of DNA repair in protection from sun damage. Ophthalmology 2013;120:13241336.

7 Goyal JL, Rao VA, Srinivasan R, Agrawal K: Oculocutaneous manifestations in xeroderma pigmentosa. Br J Ophthalmol 1994;78: 295-297.

8 Olson MT, Puttgen KB, Westra WH: Angiosarcoma arising from the tongue of an 11 -year-old girl with xeroderma pigmentosum. Head Neck Pathol 2012;6:255-257.

9 Fretzin DF, Helwig EB: Atypical fibroxanthoma of the skin. A clinicopathologic study of 140 cases. Cancer 1973;31:1541-1552.

10 Shao L, Newell B, Quintanilla N: Atypical fibroxanthoma and squamous cell carcinoma of the conjunctiva in xeroderma pigmentosum. Pediatr Dev Pathol 2007;10:149-152.

11 Taylor RW: Xeroderma pigmentosum and its relationship to malignant new-growths of the skin. Med Rec 1888;33:261-269.

12 King H, Hamilton CM: Xeroderma pigmentosum in a Negress. Arch Dermatol 1940;42: 570-575.
13 Reese AB, Wilber IB: The eye manifestations of xeroderma pigmentosum. Am J Ophthalmol 1943;26:901-911.

14 Font RL, Croxatto JO, Rao NA: Tumors of the Eye and Ocular Adnexa, ed 4. Washington, American Registry of Pathology, 2006.

15 Alfawaz AM, Al-Hussain HM: Ocular manifestations of xeroderma pigmentosum at a tertiary eye care center in Saudi Arabia. Ophthal Plast Reconstr Surg 2011;27:401-404.

16 de Feraudy S, Boubakour-Azzouz I, Fraitag S, et al: Diagnosing xeroderma pigmentosum group C by immunohistochemistry. Am J Dermatopathol 2010;32:109-117.

17 Ramkumar HL, Brooks BP, Cao X, et al: Ophthalmic manifestations and histopathology of xeroderma pigmentosum: two clinicopathological cases and a review of the literature. Surv Ophthalmol 2011;56:348-361.

18 Coroneo M: Ultraviolet radiation and the anterior eye. Eye Contact Lens 2011;37:214224.

19 DiGiovanna JJ, Kraemer KH: Shining a light on xeroderma pigmentosum. J Invest Dermatol 2012;132:785-796. 